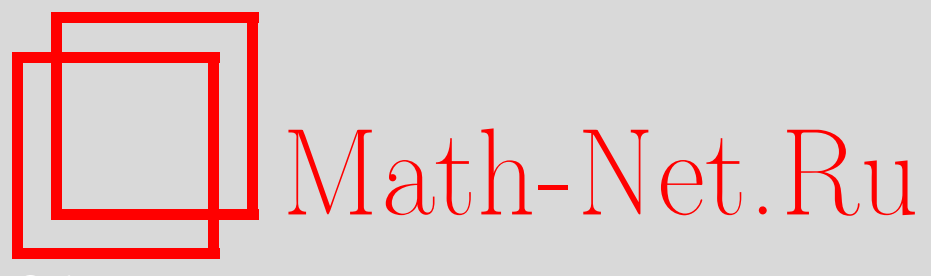

У. А. Розиков, Ш. А. Шоюсупов, Меры Гиббса для модели SOS с четырьмя состояниями на дереве Кэли, $T M \Phi$, 2006, том 149, номер 1, 18-31

DOI: https://doi.org/10.4213/tmf3825

Использование Общероссийского математического портала Math-Net.Ru подразумевает, что вы прочитали и согласны с пользовательским соглашением http://www . mathnet.ru/rus/agreement

Параметры загрузки:

IP : 54.237 .206 .68

26 апреля 2023 г., 12:39:30

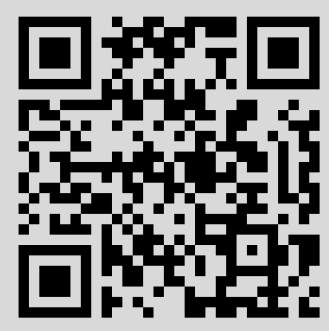




\section{МЕРЫ ГИББСА ДЛЯ МОДЕЛИ SOS С ЧЕТЫРЬМЯ СОСТОЯНИЯМИ НА ДЕРЕВЕ КЭЛИ}

\footnotetext{
Проведен анализ модели SOS (solid-on-solid) со значениями спина 0, 1, 2, 3 на дереве Кэли порядка $k \geqslant 1$. Рассмотрены трансляционно-инвариантные и периодические расщепленные меры Гиббса для этой модели. Большинство построенных нами мер Гиббса являются зеркально-симметричными.
}

Ключевые слова: дерево Кэли, конфигурация, мера Гиббса, периодическая мера Гиббса.

\section{1. ВВЕДЕНИЕ}

Дерево Кэли $\Gamma^{k}=(V, L)$ порядка $k \geqslant 1$ есть бесконечное дерево (граф без циклов), из каждой вершины которого выходит равно $k+1$ ребер, $V$ - множество вершин, $L$ - множество ребер.

Пусть $\Phi:=\{0,1, \ldots, m\}, m \geqslant 1$, и конфигурация $\sigma \in \Phi^{V}$, т.е. $\sigma=\{\sigma(x) \in \Phi$ : $x \in V\}$. Рассмотрим гамильтониан

$$
H(\sigma)=-J \sum_{\langle x, y\rangle \in L}|\sigma(x)-\sigma(y)|,
$$

где $J \in \mathbb{R}$ и $\langle x, y\rangle$ означает соседние вершины. Модель, соответствующая (1), называется моделью SOS (solid-on-solid).

Модель SOS можно рассматривать как обобщение модели Изинга [1], которая получается при $m=1$. Модель SOS имеет зеркальную симметрию, т.е. при замене $\sigma(x) \rightarrow m-\sigma(x)$ значение гамильтониана не меняется.

В работе [2] для модели SOS на $\mathbb{Z}^{d}$ доказано, что существует $T_{0}>0$ такое, что при $T<T_{0}$ структура термодинамических фаз определяется через доминирующие основные состояния модели SOS: для четных $m$ мера Гиббса (МГ) единственна, для нечетных $m$ существуют две периодические МГ.

Модель SOS на дереве Кэли рассмотрена в работе [3], где при $m=2$ найдена

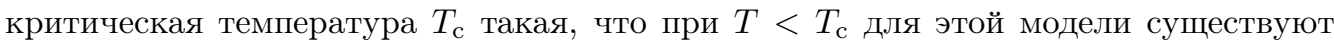
три различные трансляционно-инвариантные расщепленные МГ (ТИРМГ). Также

*Институт математики АН РУз, Ташкент, Узбекистан. E-mail: rozikovu@yandex.ru, shokir79@mail.ru 
в этой работе дано полное описание периодических МГ (ПМГ). Заметим, что при $m=2$ модель SOS совпадает с моделью Блума-Капела [4].

B работе [5] для модели SOS, используя контурный метод на дереве Кэли, мы доказали, что при достаточно низких температурах существует $m+1$ различных МГ.

В настоящей работе мы изучаем модель SOS при $m=3$, которая отличается от модели Блума-Капела. Результаты этой работы дополняют результат работы [5]: мы получим точное значение для критической температуры при $m=3$. Кроме того, мы рассмотрим периодические и непериодические МГ. Поскольку в случае $\mathbb{Z}^{d}$ число трансляционно-инвариантных мер (ТИМ) зависит от четности $m$, результаты данной работы дают возможность сравнить числа ТИРМГ для $m$ разной четности: в работе [3] рассмотрен случай $m=2$, а в нашем случае $m=3$.

\section{2. ПРЕДВАРИТЕЛЬНЫЕ СВЕДЕНИЯ}

2.1. Дерево Кэли. Известно, что дерево Кэли $\Gamma^{k}$ можно представить как свободное произведение $k+1$ циклических групп второго порядка (см., например, [6]). Обозначим эту группу как $G_{k}$. Две вершины $x, y \in V$ называются соседними, если они представляют собой концевые точки некоторого ребра $l \in L$, и в этом случае мы будем писать $l=\langle x, y\rangle$.

Расстояние $d(x, y), x, y \in V$, на дереве Кэли вводится следующим образом: $d(x, y)=d$, если $d$ есть минимальное значение, при котором найдутся вершины $x=x_{0}, x_{1}, \ldots, x_{d-1}, x_{d}=y$ такие, что $\left\langle x_{0}, x_{1}\right\rangle, \ldots,\left\langle x_{d-1}, x_{d}\right\rangle$ - соседи. Последовательность $\pi=\left\{x=x_{0}, x_{1}, \ldots, x_{d-1}, x_{d}=y\right\} \subset V$, реализующая указанный минимум, называется путем из $x$ в $y$. Положим $W_{n}=\left\{x \in V \mid d\left(x, x^{0}\right)=n\right\}, V_{n}=\bigcup_{m=0}^{n} W_{m}$, где вершина $x^{0} \in V$ фиксирована.

2.2. Пространство конфигурации и модель. Пусть спин принимает значения из множества $\Phi:=\{0,1,2,3\}$. Конфигурация $\sigma$ на $A \subseteq V$ определяется как функция $x \in A \mapsto \sigma_{A}(x) \in \Phi$; множество всех конфигураций совпадает с $\Omega_{A}=\Phi^{A}$. Положим $\Omega=\Omega_{V}$ и $\sigma=\sigma_{V}$.

Периодическая конфигурация определяется как конфигурация $\sigma \in \Omega$, которая инвариантна относительно некоторой подгруппы $G_{k}^{*} \subset G_{k}$, другими словами, конфигурация $\sigma \in \Omega$ называется периодической, если $\sigma(y x)=\sigma(x)$ для любых $x \in G_{k}$ и $y \in G_{k}^{*}$. Для данной периодической конфигурации индекс подгруппы называется периодом конфигурации. Конфигурация, инвариантная относительно всех сдвигов на дереве, называется трансляционно-инвариантной. Гамильтониан $H: \Omega \rightarrow R$ модели SOS определяется по формуле (1).

2.3. Мера Гиббса. Рассмотрим стандартную $\sigma$-алгебру $\mathcal{B}$ подмножеств $\Omega$, порожденных цилиндрическими подмножествами, и вероятностные меры на $(\Omega, \mathcal{B})$. Вероятностная мера $\mu$ называется МГ с гамильтонианом $H$, если выполняется условие Добрушина-Лэнфорда-Рюэлла

$$
\mu\left(\left\{\sigma \in \Omega: \sigma_{V_{n}}=\sigma_{n}\right\}\right)=\int_{\Omega} \mu(d \omega) \nu_{\omega_{W_{n+1}}^{V_{n}}}\left(\sigma_{n}\right)
$$


где $\nu_{\omega_{W_{n+1}}}^{V_{n}}-$ условная вероятность:

$$
\nu_{\omega_{W_{n+1}}}^{V_{n}}\left(\sigma_{n}\right)=\frac{1}{Z_{n}\left(\omega_{W_{n+1}}\right)} \exp \left(-\beta H\left(\sigma_{n} \mid \omega_{W_{n+1}}\right)\right) .
$$

Здесь

$$
\begin{gathered}
H\left(\sigma_{n} \mid \omega_{W_{n+1}}\right)=-J \sum_{\substack{\langle x, y\rangle \in L \\
x, y \in V_{n}}}\left|\sigma_{n}(x)-\sigma_{n}(y)\right|-J \sum_{\substack{\langle x, y\rangle \in L \\
x \in V_{n}, y \in W_{n+1}}}\left|\sigma_{n}(x)-\omega(y)\right|, \\
Z_{n}\left(\omega_{W_{n+1}}\right)=\sum_{\tilde{\sigma}_{n} \in \Omega_{V_{n}}} \exp \left(-\beta H\left(\tilde{\sigma}_{n} \mid \omega_{W_{n+1}}\right)\right) .
\end{gathered}
$$

Так как взаимодействуют только соседние вершины, МГ удовлетворяет свойству Маркова: если дана конфигурация $\omega_{W_{n}}$, то конфигурации в $V_{n-1}$, т.е. внутри $W_{n}$, и в $V \backslash V_{n+1}$, т.е. вне $W_{n}$, являются (условно) независимыми.

Известно, что для любого $\beta>0$ множество всех МГ образует непустое выпуклое компактное подмножество в пространстве всех вероятностных мер (см., например, [1], [7]).

Мы используем стандартное определение ТИМ (см., например, [7], [8]) и называем $\mu$ зеркально-симметричной мерой (ЗСМ), если значение этой меры сохраняется при одновременной замене $\sigma(x) \rightarrow m-\sigma(x)$ на каждой вершине $x \in V$.

2.4. Условия согласования. Зафиксируем $x^{0} \in V$. Для $x, y \in V$ будем писать $x<y$, если путь от $x^{0}$ до $y$ проходит через $x$. Вершина $y$ называется прямым потомком $x$, если $y>x$ и $x, y$ являются соседями. Через $S(x)$ обозначим множество прямых потомков $x$. Заметим, что в $\Gamma_{k}$ всякая вершина $x \neq x^{0}$ имеет $k$ прямых потомков, и $x^{0}$ имеет $k+1$ потомков.

Рассмотрим особый класс МГ, которые в работе [1] называются марковскими цепями, а в работе [3] - расщепленными МГ (РМГ).

Пусть $h: x \mapsto h_{x}=\left(h_{0, x}, h_{1, x}, \ldots, h_{m, x}\right) \in \mathbb{R}^{m+1}$ - вектор-функция от $x \in V \backslash\left\{x^{0}\right\}$. Рассмотрим вероятностное распределение $\mu^{(n)}$ на $\Omega_{V_{n}}$ :

$$
\mu^{(n)}\left(\sigma_{n}\right)=Z_{n}^{-1} \exp \left(-\beta H\left(\sigma_{n}\right)+\sum_{x \in W_{n}} h_{\sigma(x), x}\right),
$$

где $\sigma_{n} \in \Omega_{V_{n}}$,

$$
Z_{n}=\sum_{\tilde{\sigma}_{n} \in \Omega_{V_{n}}} \exp \left(-\beta H\left(\tilde{\sigma}_{n}\right)+\sum_{x \in W_{n}} h_{\tilde{\sigma}(x), x}\right) .
$$

Говорят, что вероятностное распределение $\mu^{(n)}$ согласовано, если для любых $n \geqslant 1$ и $\sigma_{n-1} \in \Omega_{V_{n-1}}$

$$
\sum_{\omega_{n} \in \Omega_{W_{n}}} \mu^{(n)}\left(\sigma_{n-1} \cup \omega_{n}\right)=\mu^{(n-1)}\left(\sigma_{n-1}\right),
$$

где $\sigma_{n-1} \cup \omega_{n} \in \Omega_{V_{n}}$. 
В этом случае существует единственная мера $\mu$ на $\Omega$ такая, что $\mu\left(\left\{\sigma_{V_{n}}=\sigma_{n}\right\}\right)=$ $\mu^{(n)}\left(\sigma_{n}\right)$ для всех $n$ и $\sigma_{n} \in \Omega_{V_{n}}$. Такая мера называется РГМ, соответствующей гамильтониану $H$ и функции $x \mapsto h_{x}, x \neq x^{0}$.

Следующее предложение дает условие на $h_{x}$, гарантирующее согласованность распределения $\mu^{(n)}\left(\sigma_{n}\right)$.

Tеорема 1 [3]. Вероятностное распределение $\mu^{(n)}\left(\sigma_{n}\right), n=1,2, \ldots$, заданное формулой (2), согласовано тогда и только тогда, когда для любого $x \in V \backslash\left\{x^{0}\right\}$ имеет место следующее уравнение:

$$
h_{x}^{*}=\sum_{y \in S(x)} F\left(h_{y}^{*}, m, \theta\right),
$$

где $\theta=e^{J \beta}, \beta=1 / T$. Здесъ $h_{x}^{*}=\left(h_{0, x}-h_{m, x}, h_{1, x}-h_{m, x}, \ldots, h_{m-1, x}-h_{m, x}\right) u$ $F(\cdot, m, \theta): \mathbb{R}^{m} \rightarrow \mathbb{R}^{m}$ - вектор-функиия, т.е.

$$
F(h, m, \theta)=\left(F_{0}(h, m, \theta), \ldots, F_{m-1}(h, m, \theta)\right), \quad h=\left(h_{0}, h_{1}, \ldots, h_{m-1}\right),
$$

такая, что

$$
F_{i}(h, m, \theta)=\ln \frac{\sum_{j=0}^{m-1} \theta^{|i-j|} e^{h_{j}}+\theta^{m-i}}{\sum_{j=0}^{m-1} \theta^{m-j} e^{h_{j}}+1}, \quad i=0, \ldots, m-1 .
$$

\section{3. ТРАНСЛЯЦИОННО-ИНВАРИАНТНЫЕ МЕРЫ}

Из теоремы 1 вытекает, что для любого $h=\left\{h_{x}, x \in V\right\}$, удовлетворяющего (4), существует единственная МГ $\mu$ (с сужением $\mu^{(n)}$, заданным формулой $\left.(2)\right)$, и наоборот. Однако анализ решения уравнения (4) для произвольного $m$ является нелегкой задачей.

В работе [3] проанализирован случай $m=2$, мы предположим, что $m=3$, т.е. $\Phi:=\{0,1,2,3\}$.

ЗАмечаниЕ 1. Поскольку в случае модели SOS на $\mathbb{Z}^{d}$ число ТИМ зависит от четности $m$, то естественно возникает вопрос: как число ТИМ будет зависеть от четности $m$ в случае модели SOS на дереве Кэли. В работе [3] рассмотрен случай четного $m=2$, а в нашем случае $m=3$, таким образом, результаты данной работы позволяют сравнить число ТИМ на дереве Кэли при различных значениях $m$.

Пусть $h_{3, x} \equiv 0$ для любого $x \in V$. При $m=3$ из (4), (5) получим

$$
\begin{aligned}
& h_{0, x}=\sum_{y \in S(x)} \ln \frac{e^{h_{0, y}}+\theta e^{h_{1, y}}+\theta^{2} e^{h_{2, y}}+\theta^{3}}{\theta^{3} e^{h_{0, y}}+\theta^{2} e^{h_{1, y}}+\theta e^{h_{2, y}}+1}, \\
& h_{1, x}=\sum_{y \in S(x)} \ln \frac{\theta e^{h_{0, y}}+e^{h_{1, y}}+\theta e^{h_{2, y}}+\theta^{2}}{\theta^{3} e^{h_{0, y}}+\theta^{2} e^{h_{1, y}}+\theta e^{h_{2, y}}+1}, \\
& h_{2, x}=\sum_{y \in S(x)} \ln \frac{\theta^{2} e^{h_{0, y}}+\theta e^{h_{1, y}}+e^{h_{2, y}}+\theta}{\theta^{3} e^{h_{0, y}}+\theta^{2} e^{h_{1, y}}+\theta e^{h_{2, y}}+1} .
\end{aligned}
$$


Естественно начать с трансляционно-инвариантного решения (6), т.е. считать, что $h_{x}=h \in \mathbb{R}^{3}$. Положим $z_{i}=e^{h_{i, x}}=e^{h_{i}}, i=0,1,2$, и $z_{3}=1$ для любого $x \in V$. Из (6) мы имеем

$$
\begin{aligned}
& z_{0}=\left(\frac{z_{0}+\theta z_{1}+\theta^{2} z_{2}+\theta^{3}}{\theta^{3} z_{0}+\theta^{2} z_{1}+\theta z_{2}+1}\right)^{k}, \\
& z_{1}=\left(\frac{\theta z_{0}+z_{1}+\theta z_{2}+\theta^{2}}{\theta^{3} z_{0}+\theta^{2} z_{1}+\theta z_{2}+1}\right)^{k}, \\
& z_{2}=\left(\frac{\theta^{2} z_{0}+\theta z_{1}+z_{2}+\theta}{\theta^{3} z_{0}+\theta^{2} z_{1}+\theta z_{2}+1}\right)^{k} .
\end{aligned}
$$

Заметим, что $z_{0}=1, z_{1}=z_{2}=z$ удовлетворяют уравнению (7а) независимо от $k, \theta$. Подставляя $z_{0}=1, z_{1}=z_{2}=z$ в (7б) и (7в), получим

$$
z=\left(\frac{\theta^{2}+(\theta+1) z+\theta}{\theta^{3}+\left(\theta^{2}+\theta\right) z+1}\right)^{k} .
$$

Введем обозначения

$$
a=\theta^{k+1}, \quad b=\frac{1-\theta+\theta^{2}}{\theta^{2}}, \quad x=\frac{z}{\theta} .
$$

ЛЕмма 1 (Ср. с леммой 10.7 в [1]). Уравнение (8) при условиях $x>0, k \geqslant 1$, $a, b>0$ имеет единственное решение, если $k=1$ или $b \leqslant\left(\frac{k+1}{k-1}\right)^{2}$. Если $k>1$ u $b>$ $\left(\frac{k+1}{k-1}\right)^{2}$, то существуют $\nu_{1}(b, k), \nu_{2}(b, k)$, удовлетворяющие условиям $0<\nu_{1}(b, k)<$ $\nu_{2}(b, k)$, такие, что уравнение имеет три решения, если $\nu_{1}(b, k)<a<\nu_{2}(b, k), u$ имеет два решения, если $a=\nu_{1}(b, k)$ или $a=\nu_{2}(b, k)$. При этом

$$
\nu_{i}(b, k)=\frac{1}{x_{i}}\left(\frac{1+x_{i}}{b+x_{i}}\right)^{k},
$$

где $x_{1}, x_{2}$ - решения уравнения $x^{2}+[2-(b-1)(k-1)] x+b=0$.

Лемма 2. Если $J \geqslant 0$, то система (7) имеет единственное решение $\left(1, z_{*}, z_{*}\right)$.

ДокАЗАтельство. Пусть $A=z_{0}+\theta z_{1}+\theta^{2} z_{2}+\theta^{3}, B=\theta^{3} z_{0}+\theta^{2} z_{1}+\theta z_{2}+1$, $A_{1}=\theta z_{0}+z_{1}+\theta z_{2}+\theta^{2}, B_{1}=\theta^{2} z_{0}+\theta z_{1}+z_{2}+\theta$. Из (7) имеем

$$
\begin{gathered}
{\left[\left(1-\theta^{3}\right) T-1\right]\left(z_{0}-1\right)+\left(\theta-\theta^{2}\right) T\left(z_{1}-z_{2}\right)=0} \\
\left.\left(\theta-\theta^{2}\right) T_{1}\left(z_{0}-1\right)+\left[(1-\theta) T_{1}-1\right]\left(z_{1}-z_{2}\right)\right]=0
\end{gathered}
$$

где $T=\left(A^{k-1}+\cdots+B^{k-1}\right) / B^{k}>0$ и $T_{1}=\left(A_{1}^{k-1}+\cdots+B_{1}^{k-1}\right) / B^{k}>0$.

Заметим, что при $\theta \geqslant 1(J \geqslant 0)$ детерминант однородной системы (10) положителен, $\Delta=(\theta-1)\left[\left(\theta^{2}-1\right) T T_{1}+\left(1+\theta+\theta^{2}\right) T+T_{1}\right]+1>0$. Следовательно, система (10) имеет единственное решение $z_{0}=1, z_{1}=z_{2}$.

Таким образом, доказано, что при $J \geqslant 0$ система (7) имеет только решения вида $(1, z, z)$. Так как $b=\frac{1-\theta+\theta^{2}}{\theta^{2}} \leqslant 1<\left(\frac{k+1}{k-1}\right)^{2}$, то в силу леммы 1 система (7) имеет единственное решение вида $\left(1, z_{*}, z_{*}\right)$. Лемма доказана. 
При $\theta \neq 1$ из системы (10) получим

СлЕДСТВИЕ. $z_{0}=1$ тогда и только тогда, когда $z_{1}=z_{2}$.

Пусть

$$
\beta_{\mathrm{c}}=\frac{1}{J} \ln \frac{2(k-1)}{k-1+\sqrt{k^{2}+14 k+1}} .
$$

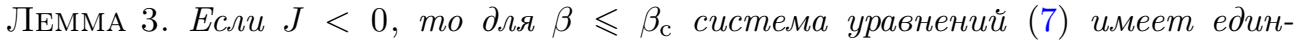
ственное решение вида $z^{*}=\left(1, z_{*}, z_{*}\right)$ (m.е. $\left.z_{0}=1\right)$, а для $\beta>\beta_{\mathrm{c}}-$ точно три решения, $z_{-}^{*}=\left(1, z_{1,-}^{*}, z_{1,-}^{*}\right), z_{0}^{*}=\left(1, z_{1,0}^{*}, z_{1,0}^{*}\right), z_{+}^{*}=\left(1, z_{1,+}^{*}, z_{1,+}^{*}\right)$, причем

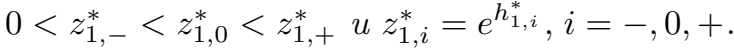

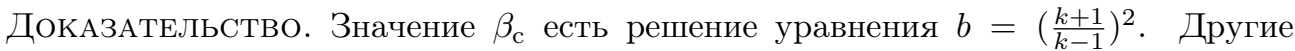
утверждения леммы 3 следуют из леммы 1.

Заметим, что $z^{*}, z_{-}^{*}, z_{0}^{*}, z_{+}^{*}$ дают зеркально-симметричное решение системы (7). Следовательно, верна

Теорема 2. Для модели SOS при $J>0$ и $m=3$ зеркально-симметричная трансляцонно-инвариантная расщепленная мера Гиббса (ЗСТИРМГ) существует и единственна для любого $\beta>0$. При $J<0$ u $m=3$, если $k \geqslant 2 u 0<\beta \leqslant \beta_{\text {c }}$, то существует единственная ЗСТИРМГ $\mu^{*} ;$ если $k \geqslant 2$ и $\beta>\beta_{\mathrm{c}}$, то существуют три ЗСТИРМГ $\mu_{-}^{*}, \mu_{0}^{*}, \mu_{+}^{*}$, соответствующие $h_{i}^{*}=\ln z_{i}^{*}, i=-, 0,+$.

Следующая лемма дает полезное свойство решения $h_{x}=\left(h_{0, x}, h_{1, x}, h_{2, x},\right)$ системы (6) при $h_{0, x} \equiv 0, h_{1, x}=h_{2, x}$.

ЛЕмма 4. Для $J<0, k \geqslant 2 u \beta>\beta_{\mathrm{c}}$, если $h_{x}=\left(0, h_{1, x}, h_{1, x}\right)$ является решением системы (6), то для любого $x \in V$

$$
z_{1,-}^{*} \leqslant z_{1, x} \leqslant z_{1,+}^{*},
$$

где $z_{1, x}=e^{h_{1, x}} u z_{-}^{*}<z_{1,+}^{*}$ определены в лемме 3 .

ДокАЗАТЕЛЬСтво. Положим $z_{x}=z_{1, x}$. Из (6) имеем

$$
z_{x}=\prod_{i=1}^{k} \frac{\theta+z_{x_{i}}}{1-\theta+\theta^{2}+\theta z_{x_{i}}}, \quad z_{x_{j}}>0, \quad j=\overline{1, k}
$$

где $x_{j}, j=\overline{1, k},-$ прямые потомки точки $x \in V$.

Пусть $\varphi(x, \theta)=(\theta+x) /\left(1-\theta+\theta^{2}+\theta x\right), \psi(x, \theta, k)=(\varphi(x, \theta))^{k}$,

$$
G\left(u_{1}, \ldots, u_{k}\right)=\prod_{i=1}^{k} \varphi\left(u_{i}, \theta\right), \quad u_{j}>0, \quad j=\overline{1, k} .
$$

Заметим, что $\psi(0, \theta, k) \leqslant G\left(u_{1}, \ldots, u_{k}\right) \leqslant \psi(\infty, \theta, k)$.

Таким образом, для $z_{x}$ имеем $\psi(0, \theta, k) \leqslant z_{x} \leqslant \psi(\infty, \theta, k)$. Теперь рассмотрим функцию $G\left(u_{1}, \ldots, u_{k}\right)$ при $\psi(0, \theta, k) \leqslant u_{j} \leqslant \psi(\infty, \theta, k)$. Тогда аналогичным образом имеем

$$
\psi(\psi(0, \theta, k), \theta, k) \leqslant z_{x} \leqslant \psi(\psi(\infty, \theta, k), \theta, k) .
$$


Итерируя эту процедуру, на $n$-м шаге получим

$$
\psi^{(n)}(0, \theta, k) \leqslant z_{x} \leqslant \psi^{(n)}(\infty, \theta, k)
$$

для любого $n \geqslant 1$ и $x \in V \backslash\left\{x^{0}\right\}$. Последовательность $\psi^{(n)}(\infty, \theta, k), n=1,2, \ldots$, убывает и ограничена снизу величиной $z_{1,+}^{*}$. Предел этой последовательности есть неподвижная точка для $\psi$, следовательно, совпадает с $z_{1,+}^{*}$. Оценка $z_{1, z}$ снизу в (11) получается аналогичным образом. Лемма доказана.

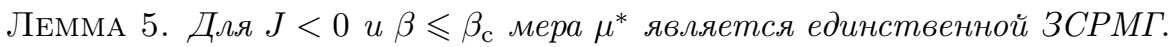

ДокАЗАтЕЛЬСтво. В условиях леммы $z_{1,-}^{*}=z_{1,+}^{*}=z_{1}^{*}$, из (11) получаем, что $h_{x}=\left(0, z_{1}^{*}, z_{1}^{*}\right)$ - единственное решение. Лемма доказана.

\section{4. ПОЛНОЕ ОПИСАНИЕ ПЕРИОДИЧЕСКИХ РМГ}

Пусть $G_{k}$ - групповое представление $\Gamma^{k}$ (см. п. 2.1).

ОПРЕдЕЛЕНИЕ 1 . Пусть $\widehat{G_{k}}-$ подгруппа группы $G_{k}$. Совокупность векторов $h=$ $\left\{h_{x}: x \in G_{k}\right\}$ назовем $\widehat{G_{k}}$-nериодической, если $h_{x}=h_{y x}$ для любых $x \in G_{k}$ и $y \in \widehat{G_{k}}$.

ОпредЕЛЕниЕ 2. Меру, соответствующую $\widehat{G_{k}}$-периодической совокупности векторов, назовем $\widehat{G_{k}}$-периодической; $G_{k}$-периодическая мера называется трансляционно-инвариантной.

ЗАмЕчаниЕ 2. Используя взаимно-однозначное соответствие между множеством вершин $V$ дерева Кэли и элементами группы $G_{k}$, в терминах самого дерева Кэли меру $\mu$ можно назвать $\widehat{G_{k}}$-периодической, если значение этой меры сохраняется при сдвиге $x \rightarrow y x$ (на дереве Кэли) для любых $x \in G_{k}, y \in \widehat{G_{k}}$. Заметим, что определение сдвига на дереве Кэли с помощью группового представления дерева Кэли является естественным.

В этом разделе мы описываем РМГ, периодические относительно любого нормального делителя конечного индекса.

Пусть $K$ - нормальный делитель индекса $r$ группы $G_{k}$, введем также обозначение $G_{k} / K=\left\{K_{0}, K_{1}, \ldots, K_{r-1}\right\}, K_{0}=K$, для факторгруппы.

Положим

$$
q_{i}(x)=\left|S(x) \cap K_{i}\right|, \quad i=0,1, \ldots, r-1 ; \quad N(x)=\left|\left\{j: q_{j}(x) \neq 0\right\}\right|,
$$

где $|\cdot|$ - число элементов множества. Пусть

$$
Q(x)=\left(q_{0}(x), q_{1}(x), \ldots, q_{r-1}(x)\right) .
$$

Заметим, что для любого $x \in G_{k}$ существует перестановка $\pi_{x}$ координат вектора $Q(x)$ такая, что

$$
\pi_{x} Q(e)=Q(x)
$$

где $e \in G_{k}$ - единичный элемент [10]. Из равенства (12) следует, что $N(x)=N(e)$ для любого $x \in G_{k} ; K$-периодическая совокупность векторов имеет следующий вид:

$$
\left\{h_{x}=h_{i} \in \mathbb{R}^{3} \text {, если } x \in K_{i}, i=0,1, \ldots, r-1\right\} .
$$


В силу (6) и равенства (12) векторы $h_{i}, i=0,1, \ldots, r-1$, удовлетворяют системе уравнений

$$
h_{m}=\sum_{j=1}^{N(e)} q_{i_{j}}(e) F\left(h_{\pi_{m}\left(i_{j}\right)} ; \theta\right)-F\left(h_{\pi_{m}\left(i_{j}\right)} ; \theta\right),
$$

где $m=\overline{0, r-1}, j_{0}=\overline{1, N(e)}, N(e)=\left|\left\{i_{1}, i_{2}, \ldots, i_{N(e)}\right\}\right|$.

Пусть функция $F: R^{4} \rightarrow R^{4}, F(h, \theta)=\left(F_{0}(h, \theta), F_{1}(h, \theta), F_{2}(h, \theta)\right)$, задается равенствами

$$
\begin{aligned}
& F_{0}(h, \theta)=\ln \frac{e^{h_{0}}+\theta e^{h_{1}}+\theta^{2} e^{h_{2}}+\theta^{3}}{\theta^{3} e^{h_{0}}+\theta^{2} e^{h_{1}}+\theta e^{h_{2}}+1}, \\
& F_{1}(h, \theta)=\ln \frac{\theta e^{h_{0}}+e^{h_{1}}+\theta e^{h_{2}}+\theta^{2}}{\theta^{3} e^{h_{0}}+\theta^{2} e^{h_{1}}+\theta e^{h_{2}}+1}, \\
& F_{2}(h, \theta)=\ln \frac{\theta^{2} e^{h_{0}}+\theta e^{h_{1}}+e^{h_{2}}+\theta}{\theta^{3} e^{h_{0}}+\theta^{2} e^{h_{1}}+\theta e^{h_{2}}+1} .
\end{aligned}
$$

Лемма 6. Если $\theta \neq 1$, то $F(h, \theta)=F(l, \theta)$ тогда и только тогда, когда $h=l$.

ДоказАтельство. Необходимость. Пусть $F(h, \theta)=F(l, \theta)$, тогда

$$
\begin{aligned}
& {\left[1+\theta^{2}+\theta^{4}+\theta^{2} t_{1}+\theta\left(1+\theta^{2}\right) t_{2}\right]\left(z_{0}-t_{0}\right)+} \\
& \quad+\left[\theta^{2}\left(t_{2}-t_{0}\right)+\theta\left(1+\theta^{2}\right)\right]\left(z_{1}-t_{1}\right)+\theta\left[\theta-\left(1+\theta^{2}\right) t_{0}-\theta t_{1}\right]\left(z_{2}-t_{2}\right)=0, \\
& {\left[\theta^{2} t_{2}+\theta\left(1+\theta^{2}\right)\right]\left(z_{0}-t_{0}\right)+\left[1+\theta^{2}+\theta t_{2}\right]\left(z_{1}-t_{1}\right)+\theta\left[1-t_{1}-\theta t_{2}\right]\left(z_{2}-t_{2}\right)=0,} \\
& \theta^{2}\left(z_{0}-t_{0}\right)+\theta\left(z_{1}-t_{1}\right)+\left(z_{2}-t_{2}\right)=0,
\end{aligned}
$$

где $z_{i}=e^{h_{i}}, t_{i}=e^{l_{i}}, i=0,1,2$. Заметим, что детерминант однородной системы (15) положителен при $\theta \neq 1, \Delta=\left(1+\theta^{3} t_{0}+\theta^{2} t_{1}+\theta t_{2}\right)^{2}>0$. Следовательно, (15) имеет единственное решение $z_{i}=t_{i}, i=0,1,2$.

Достаточность очевидна. Лемма доказана.

Пусть $G_{k}^{(2)}$ - подгруппа группы $G_{k}$, состоящая из слов четной длины. Очевидно, что $G_{k}^{(2)}$ является подгруппой индекса два.

Теорема 3. Для любого нормального делителя конечного индекса $K$ всякая $K$ периодическая МГ совпадает либо с ТИМ, либо с $G_{k}^{(2)}$-периодической мерой.

ДокАЗАТЕЛЬСтво. Используя (13), получим

$$
F\left(h_{\pi\left(i_{1}\right)}, \theta\right)=F\left(h_{\pi\left(i_{2}\right)}, \theta\right)=\cdots=F\left(h_{\pi\left(i_{N(e)}\right)}, \theta\right) .
$$

Отсюда в силу леммы 6 имеем

$$
h_{\pi_{m}\left(i_{1}\right)}=h_{\pi_{m}\left(i_{2}\right)}=\cdots=h_{\pi_{m}\left(i_{N(e)}\right)} .
$$

Следовательно, $h_{x}=h_{y}=h_{1}$, если $x, y \in S_{1}(z), z \in G_{k}^{(2)} ; h_{x}=h_{y}=h_{2}$, если $x, y \in S_{1}(z), z \in G_{k} \backslash G_{k}^{(2)}$, где $S_{1}(x)=\{y \in V:\langle x, y\rangle\}$. Таким образом, если $h_{1}=h_{2}$, то $h_{x}$ - постоянная функция; если $h_{1} \neq h_{2}$, то функция $h_{x}$ является $G_{k}^{(2)}$ периодической. Теорема доказана. 
Пусть $K$ - нормальный делитель конечного индекса группы $G_{k}$. Возникает вопрос, при каком условии на $K$ всякая $K$-периодическая мера Гиббса является ТИМ? Пусть $I(K)=K \cap\left\{a_{1}, \ldots, a_{k+1}\right\}$.

Теорема 4. Если $I(K) \neq \varnothing$, то всякая $K$-периодическая $М Г$ для модели $S O S$ является ТИМ.

ДоКАЗАТЕЛЬСТво. Если $x \in K$, то для того чтобы $x a_{i} \in K$, необходимо и достаточно, чтобы $a_{i} \in K$. Так как $I(K) \neq \varnothing$, то существует $a_{i} \in K$. Следовательно, $K$ содержит подмножество $K a_{i}=\left\{x a_{i}: x \in K\right\}$. В силу теоремы 3 имеем $h_{x}=h_{1}$ и $h_{x a_{i}}=h_{2}$. Поскольку $x$ и $x a_{i}$ принадлежат классу $K$, то $h_{x}=h_{x a_{i}}=h_{1}=h_{2}$. Теорема доказана.

В силу теорем 3,4 задача описания $K$-периодических МГ при $I(K) \neq \varnothing$ сводится к описанию неподвижных точек отображения

$$
h=k F(h ; \theta),
$$

а при $I(K)=\varnothing$ сводится к описанию решения системы

$$
\begin{aligned}
h & =k F(l ; \theta), \\
l & =k F(h ; \theta) .
\end{aligned}
$$

Заметим, что отображение (16) описывает ТИМ (см. (7)). Система (17) описывает периодические меры с периодом два, точнее, $G_{k}^{(2)}$-периодические меры.

Из (17) получим

$$
\begin{aligned}
& z_{0}=\left(\frac{t_{0}+\theta t_{1}+\theta^{2} t_{2}+\theta^{3}}{\theta^{3} t_{0}+\theta^{2} t_{1}+\theta t_{2}+1}\right)^{k}, \\
& z_{1}=\left(\frac{\theta t_{0}+t_{1}+\theta t_{2}+\theta^{2}}{\theta^{3} t_{0}+\theta^{2} t_{1}+\theta t_{2}+1}\right)^{k}, \\
& z_{2}=\left(\frac{\theta^{2} t_{0}+\theta t_{1}+t_{2}+\theta}{\theta^{3} t_{0}+\theta^{2} t_{1}+\theta t_{2}+1}\right)^{k}, \\
& t_{0}=\left(\frac{z_{0}+\theta z_{1}+\theta^{2} z_{2}+\theta^{3}}{\theta^{3} z_{0}+\theta^{2} z_{1}+\theta z_{2}+1}\right)^{k}, \\
& t_{1}=\left(\frac{\theta z_{0}+z_{1}+\theta z_{2}+\theta^{2}}{\theta^{3} z_{0}+\theta^{2} z_{1}+\theta z_{2}+1}\right)^{k}, \\
& t_{2}=\left(\frac{\theta^{2} z_{0}+\theta z_{1}+z_{2}+\theta}{\theta^{3} z_{0}+\theta^{2} z_{1}+\theta z_{2}+1}\right)^{k} .
\end{aligned}
$$

Лемма 7. Для $J<0(\theta<1)$ система (18) верна только при $z_{i}=t_{i}, i=0,1,2$. 
ДокАЗАтЕЛЬСтво. Пусть $u_{i}=z_{i}^{1 / k}, v_{i}=t_{i}^{1 / k}, i=0,1,2$. Тогда из (18) получим

$$
\begin{gathered}
{\left[A+B_{0}\left(1+\theta^{2}+\theta^{4}+\theta^{2} u_{1}^{k}+\theta\left(1+\theta^{2}\right) u_{2}^{k}\right)\right]\left(u_{0}-v_{0}\right)+} \\
+\left[B_{1}\left(\theta^{2}\left(u_{2}^{k}-u_{0}^{k}\right)+\theta^{3}+\theta\right)\right]\left(u_{1}-v_{1}\right)+ \\
+\left[B_{2}\left(\theta^{2}-\left(\theta^{3}+\theta\right) u_{0}^{k}-\theta^{2} u_{1}^{k}\right)\right]\left(u_{2}-v_{2}\right)=0, \\
{\left[B_{0}\left(\theta^{2} u_{2}^{k}+\theta^{3}+\theta\right)\right]\left(u_{0}-v_{0}\right)+\left[A+B_{1}\left(\theta u_{2}^{k}+\theta^{2}+1\right)\right]\left(u_{1}-v_{1}\right)+} \\
+\left[B_{2}\left(\theta-\theta u_{1}^{k}-\theta^{2} u_{0}^{k}\right)\right]\left(u_{2}-v_{2}\right)=0, \\
\theta^{2} B_{0}\left(u_{0}-v_{0}\right)+\theta B_{1}\left(u_{1}-v_{1}\right)+\left(A+B_{2}\right)\left(u_{2}-v_{2}\right)=0,
\end{gathered}
$$

где

$$
\begin{gathered}
A=\left(\theta^{3} v_{0}^{k}+\theta^{2} v_{1}^{k}+\theta v_{2}^{k}+1\right)\left(\theta^{3} u_{0}^{k}+\theta^{2} u_{1}^{k}+\theta u_{2}^{k}+1\right)\left(1-\theta^{2}\right)^{-1}>0, \\
B_{i}=u_{i}^{k-1}+\cdots+v_{i}^{k-1}>0, \quad i=0,1,2 .
\end{gathered}
$$

Нетрудный, но достаточно громоздкий анализ показывает, что детерминант системы (19) положителен, следовательно, $z_{i}=t_{i}, i=0,1,2$. Лемма доказана.

Теперь рассмотрим антиферромагнитный случай $J>0(\theta>1)$. В этом случае в силу леммы 2 система (18) имеет единственное решение $\left(1, z_{1}^{*}, z_{1}^{*}, 1, t_{1}^{*}, t_{1}^{*}\right)$.

Пусть $z_{0}=t_{0}=1, z_{1}=z_{2}=z, t_{1}=t_{2}=t$, тогда из (18) имеем

$$
\begin{aligned}
& z=\left(\frac{\theta+t}{1-\theta+\theta^{2}+\theta t}\right)^{k} \\
& t=\left(\frac{\theta+z}{1-\theta+\theta^{2}+\theta z}\right)^{k}
\end{aligned}
$$

Лемма 8. Пусть $\left(z_{*}, z_{*}\right)$-решение системь (20). Если

$$
\frac{k z_{*}(\theta-1)}{\left(\theta+z_{*}\right)\left(1-\theta+\theta^{2}+\theta z_{*}\right)}>1
$$

то система (10) имеет три решения $\left(z_{-}^{*}, z_{+}^{*}\right),\left(z_{*}, z_{*}\right),\left(z_{+}^{*}, z_{-}^{*}\right)$, где $z_{-}^{*}=\psi\left(z_{+}^{*}, \theta, k\right)$ $u$

$$
\psi(x, \theta, k)=\left(\frac{\theta+x}{1-\theta+\theta^{2}+\theta x}\right)^{k} .
$$

ДокАЗАтЕльство. Если справедливо неравенство (21), то решение $z_{*}-$ неустойчивая точка для $\psi$. Для любого $z \geqslant 1$ итерации $\psi^{(2 n)}(z, \theta, k)>z_{*}$ монотонно убывают, следовательно, существует $\lim _{n \rightarrow \infty} \psi^{(2 n)}(z, \theta, k)=z_{+}^{*} \geqslant z_{*}$, который является решением уравнения

$$
z=\psi(\psi(z, \theta, k), \theta, k)
$$

Так как $z^{*}-$ неустойчивая точка, $z_{+}^{*}>z_{*}$. Аналогично $z_{-}^{*}=\psi\left(z_{+}^{*}, \theta, k\right)<z_{*}$, что также является решением (22). Лемма доказана.

Таким образом, доказана 
ТеОРема 5. Для модели SOS при $m=3$ на дереве Кэли относительно любого нормального делителя конечного индекса $K$ справедливы следующие утверждения:

1) в ферромагнитном случае $(J<0)$ и при $J=0 \quad K$-периодическая МГ совпадает c ТИМГ;

2) в антиферромагнитном случае $(J>0)$ :

а) если $I(K) \neq \varnothing$, то $K$-периодическая МГ совпадает с ТИМГ;

б) если выполнено неравенство (21) и $I(K)=\varnothing$, то существуют три K-периодические $М Г \mu_{12}, \mu_{*}, \mu_{21}$.

При этом $\mu_{*}$ есть ТИМ, а $\mu_{12}$ и $\mu_{21}$ - периодические меры с периодом два, т.е. $G_{k}^{(2)}$-периодические меры.

\section{5. НЕПЕРИОДИЧЕСКИЕ РМГ}

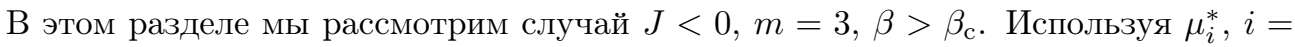
$-, 0,+$, покажем, что система (6) имеет несчетное число непериодических решений.

Пусть дан бесконечный путь $\pi=\left\{x^{0}=x_{0}<x_{1}<x_{2}<\cdots\right\}$ на дереве Кэли $\Gamma^{k}$. Этому пути стандартным образом [11], [12] можно поставить в соответствие $t \in[0,1]$. Пусть $\pi_{1}=\left\{x_{0}, x_{1}, \ldots\right\}$ и $\pi_{2}=\left\{y_{0}, y_{1}, \ldots\right\}$ - два бесконечных пути с $x_{0}=y_{0}=x^{0}$. Мы отображаем пару $\left(\pi_{1}, \pi_{2}\right)$ в вектор-функцию $h^{\pi_{1} \pi_{2}}: x \in V \mapsto h_{x}^{\pi_{1} \pi_{2}}$, удовлетворяющую (6).

Пути $\pi_{1}$ и $\pi_{2}$ разбивают $\Gamma^{k}$ на три подграфа $\Gamma_{1}^{k}, \Gamma_{2}^{k}, \Gamma_{3}^{k}$, если $\pi_{1} \neq \pi_{2}$, и на два подграфа $\Gamma_{1}^{k}, \Gamma_{3}^{k}$, если $\pi_{1}=\pi_{2}$.

Вектор-функция $h^{\pi_{1} \pi_{2}}$ задается следующим образом:

$$
h_{x}^{\pi_{1} \pi_{2}}= \begin{cases}h_{-}^{*}, & \text { если } x \in \Gamma_{1}^{k}, \\ h_{0}^{*}, & \text { если } x \in \Gamma_{2}^{k}, \\ h_{+}^{*}, & \text { если } x \in \Gamma_{3}^{k},\end{cases}
$$

векторы $h_{i}^{*}=\left(0, \ln z_{1, i}^{*}, \ln z_{1, i}^{*}\right), i=-, 0,+$, определяются решениями системы $(7)$.

Пусть $h=\left(h_{0}, h_{1}, h_{2}\right) \in \mathbb{R}^{3}$,

$$
\|h\|=\max \left\{\left|h_{0}\right|,\left|h_{1}\right|,\left|h_{2}\right|\right\}
$$

функция $h \mapsto F(h)$ определена равенствами (14). Введем обозначение

$$
f(x, y, z) \equiv f\left(x, y, z, a_{1}, a_{2}, a_{3}, a_{4}, b_{1}, b_{2}, b_{3}, b_{4}\right)=\ln \frac{a_{1} e^{x}+a_{2} e^{y}+a_{3} e^{z}+a_{4}}{b_{1} e^{x}+b_{2} e^{y}+b_{3} e^{z}+b_{4}},
$$

где

$$
a_{i} \geqslant 0, \quad b_{i} \geqslant 0, \quad i=1,2,3,4, \quad a_{1}+a_{2}+a_{3}+a_{4}>0, \quad b_{1}+b_{2}+b_{3}+b_{4}>0 .
$$


Лемма 9. Имеют место следующие оценки:

a) если $a_{1} a_{2} a_{3} a_{4} b_{1} b_{2} b_{3} b_{4}>0$, mo

$$
\begin{aligned}
& \left|\frac{\partial f}{\partial x}\right| \leqslant \max \left\{\frac{\left|a_{1} b_{2}-b_{1} a_{2}\right|}{\left(\sqrt{a_{1} b_{2}}+\sqrt{b_{1} a_{2}}\right)^{2}}, \quad \frac{\left|a_{1} b_{3}-b_{1} a_{3}\right|}{\left(\sqrt{a_{1} b_{3}}+\sqrt{b_{1} a_{3}}\right)^{2}}, \quad \frac{\left|a_{1} b_{4}-b_{1} a_{4}\right|}{\left(\sqrt{a_{1} b_{4}}+\sqrt{b_{1} a_{4}}\right)^{2}}\right\}, \\
& \left|\frac{\partial f}{\partial y}\right| \leqslant \max \left\{\frac{\left|a_{1} b_{2}-b_{1} a_{2}\right|}{\left(\sqrt{a_{1} b_{2}}+\sqrt{b_{1} a_{2}}\right)^{2}}, \quad \frac{\left|a_{2} b_{3}-b_{2} a_{3}\right|}{\left(\sqrt{a_{2} b_{3}}+\sqrt{b_{2} a_{3}}\right)^{2}}, \quad \frac{\left|a_{2} b_{4}-b_{2} a_{4}\right|}{\left(\sqrt{a_{2} b_{4}}+\sqrt{b_{2} a_{4}}\right)^{2}}\right\}, \\
& \left|\frac{\partial f}{\partial z}\right| \leqslant \max \left\{\frac{\left|a_{1} b_{3}-b_{1} a_{3}\right|}{\left(\sqrt{a_{1} b_{3}}+\sqrt{b_{1} a_{3}}\right)^{2}}, \quad \frac{\left|a_{2} b_{3}-b_{2} a_{3}\right|}{\left(\sqrt{a_{2} b_{3}}+\sqrt{b_{2} a_{3}}\right)^{2}}, \quad \frac{\left|a_{3} b_{4}-b_{3} a_{4}\right|}{\left(\sqrt{a_{3} b_{4}}+\sqrt{b_{3} a_{4}}\right)^{2}}\right\} ;
\end{aligned}
$$

б) если $a_{1} a_{2} a_{3} a_{4} b_{1} b_{2} b_{3} b_{4}=0$, mo

$$
\left|\frac{\partial f}{\partial x}\right| \leqslant 1, \quad\left|\frac{\partial f}{\partial y}\right| \leqslant 1, \quad\left|\frac{\partial f}{\partial z}\right| \leqslant 1 .
$$

ДокАЗАтЕЛЬСтво. Пусть $a, b, c, d \geqslant 0, a+b>0, c+d>0$. Введем обозначение $f_{1}(x)=\ln \frac{a e^{x}+b}{c e^{x}+d}$. Легко проверить, что

$$
\left|f_{1}^{\prime}(x)\right| \leqslant \begin{cases}\frac{|a d-b c|}{(\sqrt{a d}+\sqrt{b c})^{2}}, & \text { если } a b c d \neq 0 \\ 1, & \text { если } a b c d=0\end{cases}
$$

Рассмотрим $f_{2}(x, y)=\ln \frac{a_{1} e^{x}+a_{2} e^{y}+a_{3}}{b_{1} e^{x}+b_{2} e^{y}+b_{3}}$. В силу неравенства $(26)$

$$
\left|\frac{\partial f_{2}(x, y)}{\partial x}\right| \leqslant \frac{\left|a_{1}\left(b_{2} e^{y}+b_{3}\right)-b_{1}\left(a_{2} e^{y}+a_{3}\right)\right|}{\left(\sqrt{a_{1}\left(b_{2} e^{y}+b_{3}\right)}+\sqrt{b_{1}\left(a_{2} e^{y}+a_{3}\right)}\right)^{2}} .
$$

Пусть $t=e^{y}, \varphi(t)=\frac{\left|a_{1}\left(b_{2} t+b_{3}\right)-b_{1}\left(a_{2} t+a_{3}\right)\right|}{\left(\sqrt{a_{1}\left(b_{2} t+b_{3}\right)}+\sqrt{b_{1}\left(a_{2} t+a_{3}\right)}\right)^{2}}$. Тогда

$$
\varphi^{\prime}(t)=\sqrt{\frac{a_{1} b_{1}}{\left(a_{2} t+a_{3}\right)\left(b_{2} t+b_{3}\right)}} \cdot \frac{a_{3} b_{2}-a_{2} b_{3}}{\left(\sqrt{a_{1}\left(b_{2} t+b_{3}\right)}+\sqrt{b_{1}\left(a_{2} t+a_{3}\right)}\right)^{2}} .
$$

Следовательно, $\varphi(t)$ монотонно возрастает при $a_{3} b_{2}-a_{2} b_{3} \geqslant 0$ и монотонно убывает при $a_{3} b_{2}-a_{2} b_{3}<0$.

Так как $t>0$, из (27) имеем

$$
\frac{\partial f_{2}(x, y)}{\partial x} \leqslant \max \{|\varphi(0)|,|\varphi(\infty)|\}=\max \left\{\frac{\left|a_{1} b_{3}-b_{1} a_{3}\right|}{\left(\sqrt{a_{1} b_{3}}+\sqrt{b_{1} a_{3}}\right)^{2}}, \frac{\left|a_{1} b_{2}-b_{1} a_{2}\right|}{\left(\sqrt{a_{1} b_{2}}+\sqrt{b_{1} a_{2}}\right)^{2}}\right\} .
$$

Используя (28), аналогичным образом для $\partial f(x, y, z) / \partial x$ получим первое неравенство пункта "а" леммы 9. Остальные два неравенства доказываются аналогично. Неравенства пункта "б" получаются из неравенств пункта "а", если хотя бы один из параметров $a_{1}, a_{2}, a_{3}, a_{4}, b_{1}, b_{2}, b_{3}, b_{4}$ равен нулю и при этом имеют место неравенства (25). Лемма доказана.

Заметим, что каждая координата функции $F$ (см. (14)) имеет вид (24). Из леммы 9 вытекает 
Лемма 10. Для любого $h=\left(h_{0}, h_{1}, h_{2}\right) \in \mathbb{R}^{3}$ имеют место оценки

$$
\begin{gathered}
\left|\frac{\partial F_{0}}{\partial h_{j}}\right| \leqslant \frac{\left|1-\theta^{3}\right|}{1+\theta^{3}}, \quad\left|\frac{\partial F_{1}}{\partial h_{j}}\right| \leqslant \frac{\left|1-\theta^{2}\right|}{1+\theta^{2}}, \quad\left|\frac{\partial F_{2}}{\partial h_{j}}\right| \leqslant \frac{|1-\theta|}{1+\theta} ; \\
\|F(h, \theta)-F(l, \theta)\| \leqslant 3 \frac{\left|1-\theta^{3}\right|}{1+\theta^{3}}\|h-l\|, \quad h, l \in \mathbb{R}^{3} .
\end{gathered}
$$

ДокАЗАтЕльство. Неравенства (29а) суть следствия неравенств леммы 9. Для доказательства (29б) запишем

$$
\begin{aligned}
\|F(h)-F(l)\| & =\max _{i=0,1,2}\left\{\left|F_{i}(h)-F_{i}(l)\right|\right\} \leqslant \\
& \leqslant \max _{i=0,1,2}\left\{\left|\frac{\partial F_{i}}{\partial h_{0}}\right|\left|h_{0}-l_{0}\right|+\left|\frac{\partial F_{i}}{\partial h_{1}}\right|\left|h_{1}-l_{1}\right|+\left|\frac{\partial F_{i}}{\partial h_{2}}\right|\left|h_{2}-l_{2}\right|\right\} \leqslant \\
& \leqslant 3 \frac{\left|1-\theta^{3}\right|}{1+\theta^{3}}\|h-l\| .
\end{aligned}
$$

Используя лемму 10 (см. аналогичные рассуждения по поводу теоремы 3 в работе [12]), можно доказать следующую теорему.

ТЕОрема 6. Для любых бесконечных путей $\pi_{1}, \pi_{2}$ существует единственная вектор-функиия $h^{\pi_{1} \pi_{2}}$, удовлетворяющая соотношениям (6) и (23).

Отобразим $\left(\pi_{1}, \pi_{2}\right)$ в пару $(t, s) \in[0,1] \times[0,1]$. Можно доказать (см. [11]-[13]), что $h^{\pi_{1}(t) \pi_{2}(s)}$ различны при разных $(t, s) \in D=\left\{(u, v) \in[0,1]^{2}: u \leqslant v\right\}$.

По теореме 1 каждой совокупности $h^{\pi_{1}(t) \pi_{2}(s)}$ можно сопоставить РМГ на $\Gamma^{k}$, которую мы обозначим через $\mu(t, s)$. Таким образом, доказана

Теорема 7. Для любого $(t, s) \in D$ существует единственная РМГ $\mu(t, s)$. Более того, $\mu(0,0)=\mu_{+}^{*}, \mu(0,1)=\mu_{0}^{*}, \mu(1,1)=\mu_{-}^{*}$.

\section{6. ОБСУЖДЕНИЕ}

Описание множества всех соответствующих гамильтониану (1) гиббсовских распределений является одной из основных задач. Но полностью решить ее пока не удается даже для достаточно простых гамильтонианов (см., например, [6], [10]-[14]).

Простота дерева Кэли позволяет описать достаточно широкий класс МГ для моделей без "хороших" симметрий [9], [15] и с конкурирующими взаимодействиями (см., например, [16]).

Результаты настоящей работы дают возможность указать явно значения $\beta$, при которых существуют периодические МГ. С другой стороны, имея в виду результаты работы [3] и настоящей работы, можно заметить, что число ТИРМГ одинаково и равно трем при $m=2$ и $m=3$. Это демонстрирует существенное различие между моделями SOS на $\mathbb{Z}^{d}$ и $\Gamma^{k}$, так как в случае $\mathbb{Z}^{d}$ число ТИМ зависит от четности $m$ (см. [2]): для четных $m$ МГ единственна, для нечетных $m$ существуют две периодические МГ. 
Благодарности. У. Розиков благодарит Университет La Sapienza (Рим, Италия) за неоднократные поддержки поездок в 2005 г., а также профессоров G. Gallavotti и M. Cassandro за приглашения в "La Sapienza". Работа частично поддержана NATO (Reintegration Grant: Fel. RIG. 980771) и Центром по науке и технологиям Республики Узбекистан (грант Ф.-1.1.2). Авторы выражают благодарность рецензенту за полезные замечания по работе.

\section{Список литературы}

[1] К. Престон, Гиббсовские состояния на счетных множествах, Мир, М., 1977.

[2] A. E. Mazel, Yu. M. Suhov, J. Statist. Phys., 64 (1991), 111-134.

[3] U.A. Rozikov, Yu.M. Suhov, Gibbs measures for SOS models on a Cayley tree, math.PR/0409047.

[4] R. Fernandez, Contour ensembles and the description of Gibbsian probability distributions at low temperature, http://www.univ-rouen.fr//LMRS/Persopage/Fernandez/ resucont.html, 1998.

[5] U. A. Rozikov, Lett. Math. Phys., 71:1 (2005), 27-38.

[6] Н. Н. Ганиходжаев, У. А. Розиков, ТМФ, 111:1 (1997), 109-117.

[7] Я. Г. Синай, Теория фазовых переходов: строгие результаты, Наука, М., 1980.

[8] H. O. Georgii, Gibbs Measures and Phase Transitions, De Gruyter, Berlin, 1988.

[9] Yu. M. Suhov, U. A. Rozikov, Queueing Syst., 46 (2004), 197-212.

[10] У.А. Розиков, ТМФ, 112:1 (1997), 170-175.

[11] П. М. Блехер, Н.Н.Ганиходжаев, Теория вер. примен., 35:2 (1990), 220-230.

[12] У.А. Розиков, ТМФ, 118:1 (1999), 95-104.

[13] У.А. Розиков, Сибирск. матем. журнал, 39:2 (1998), 427-435.

[14] Н. Н. Ганиходжаев, ТМФ, 85:2 (1990), 163-175.

[15] J. B. Martin, U. A. Rozikov, Yu. M. Suhov, J. Nonlinear Math. Phys., 12:3 (2005), 432-448.

[16] F. M. Mukhamedov, U. A. Rozikov, J. Statist. Phys., 114 (2004), 825-848; 119 (2005), $427-446$.

Поступила в редакцию 16.01.2006, после доработки 29.03.2006 